\title{
A Novel Approach to the Synthesis of $\alpha$-Aminonitriles Using Triphenyl- phosphine Dibromide under Solvent-Free Conditions
}

\author{
Devdutt Chaturvedi, ${ }^{* a}$ Amit K. Chaturvedi, ${ }^{b}$ Parmesh K. Dwivedi, ${ }^{a}$ Nisha Mishra ${ }^{\mathrm{b}}$ \\ a Laboratory of Medicinal Chemistry, Amity Institute of Pharmacy, Amity University Uttar Pradesh, Lucknow Campus, Lucknow 226028, \\ U. P., India \\ Fax+91(522)2399610; E-mail: devduttchaturvedi@gmail.com; E-mail: dchaturvedi@1ko.amity.edu \\ b Synthetic Research Laboratory, Department of Chemistry, B. S. A. P. G. College, Mathura 281004, U. P., India \\ Received: 06.08.2012; Accepted after revision: 02.11.2012
}

\begin{abstract}
A quick and highly efficient, one-pot, three-component, solvent-free method for the synthesis of $\alpha$-aminonitriles starting from the corresponding carbonyl compounds, amines, and trimethylisocyanide using triphenylphosphine dibromide, has been developed. Diverse $\alpha$-aminonitriles have been synthesized in good to excellent yields (80-99\%) using a range of aldehydes, ketones and amines.
\end{abstract}

Key words: multicomponent reactions, Strecker synthesis, aminonitriles, carbonyl compounds

$\alpha$-Aminonitriles constitute a major class of naturally occurring compounds that display remarkable biological activities $^{1}$ (anticancer, antibacterial, antifungal, antibiotic, and antiviral) and also serve as efficient precursors for the synthesis of natural and unnatural $\alpha$-amino acids. ${ }^{2}$ They have also been widely used as essential building blocks in peptide and protein synthesis. ${ }^{3}$ Their synthetic utility has further been applied as a versatile synthon for the syntheses of amides, diamines, and various kinds of structurally diverse nitrogen and sulfur heterocycles ${ }^{4}$ such as imidazoles and thiadiazoles. Furthermore, their synthetic utility has also been extended through carbanion-induced nucleophilic attack on the $\alpha$-carbon atom with a variety of electrophiles, offering the possibility of further synthetic transformations. ${ }^{5}$

Among the reported methods, nucleophilic addition of cyanide ion to imines (Strecker reaction) offers one of the most direct approaches to the synthesis of $\alpha$-aminonitriles. ${ }^{6}$ The cyanide sources used during the course of this reaction include $\mathrm{HCN}, \mathrm{KCN}$, TMSCN, $(\mathrm{EtO})_{2} \mathrm{P}(\mathrm{O}) \mathrm{CN}$, $\mathrm{Et}_{2} \mathrm{AlCN}, \mathrm{Bu}_{3} \mathrm{SnCN}, \mathrm{MeCOCN}$, acetone cyanohydrin, acyl cyanides, ethyl cyanoformate, bis(dialkyl)aminocyanoboranes, and $\mathrm{K}_{4}\left[\mathrm{Fe}(\mathrm{CN})_{6}\right]{ }^{7}$ the majority of which are hazardous, toxic, and require harsh reaction conditions. In recent years, in the search for novel and efficient protocols for the synthesis of $\alpha$-aminonitriles, a broad spectrum of metal complexes, Lewis acids, solid acids, bases, and organic catalysts have been developed to promote this reaction. ${ }^{8,9}$ The majority of these catalysts are only efficient for the synthesis of $\alpha$-aminonitriles from active aldehydes and are not suitable for ketone substrates.

SYNLETT 2013, 24, 0033-0036

Advanced online publication: 04.12.2012

DOI: 10.1055/s-0032-1317690; Art ID: ST-2012-D0664-L

(c) Georg Thieme Verlag Stuttgart · New York
Therefore, there is continuing interest in developing new, efficient and safer protocols employing mild reaction conditions.

In recent years, triphenylphosphine dibromide $\left(\mathrm{Ph}_{3} \mathrm{PBr}_{2}\right)$ has emerged as a versatile reagent in organic synthesis. ${ }^{10}$ Our group has been engaged in the development of novel and efficient synthetic methodologies. ${ }^{11}$ In the present communication, we wish to report an efficient method for the synthesis of $\alpha$-aminonitriles through reaction of the corresponding carbonyl compounds, amines, and TMSCN using a catalytic amount of triphenylphosphine dibromide (TPPDB) under solvent-free conditions. ${ }^{12}$ Triphenylphosphine dibromide was synthesized by the reported procedure. ${ }^{10}$

To optimize the protocol, the reaction of an equimolar amount of aniline, benzaldehyde, and trimethysilylcyanide, using a catalytic amount of TPPDB, was studied in a range of anhydrous solvents $\left(\mathrm{CH}_{2} \mathrm{Cl}_{2}, \mathrm{THF}, \mathrm{Et}_{2} \mathrm{O}\right.$, $\mathrm{MeCN}, \mathrm{DMF}, \mathrm{MeNO}_{2}$, and $\mathrm{MeOH}$ ) at room temperature and the corresponding $\alpha$-aminonitrile was isolated. The best yields $(99 \%)$ of the desired $\alpha$-aminonitrile were achieved using at least $10 \mathrm{~mol} \%$ TPPDB in the absence of solvent (Scheme 1).

$$
\begin{aligned}
& \mathrm{R}_{\mathrm{R}^{2}}+\mathrm{R}^{3} \mathrm{NH}_{2}+\mathrm{TMSCN}_{\substack{\text { solvent-free, r.t. } \\
80-99 \%}} \\
& \mathrm{R}^{1}=\text { alkyl, cycloalkyl, aryl, } \\
& \begin{array}{l}
\text { naphthyl, anthracenyl, heteroaryl } \\
\mathrm{R}^{2}=\mathrm{H}, \mathrm{Me}, \mathrm{Et}, \mathrm{Ph} \\
\mathrm{R}^{3}=\text { alkyl, } \mathrm{Ph}, \text { aryl }
\end{array}
\end{aligned}
$$

Scheme 1

It was subsequently observed that the desired $\alpha$-aminonitrile could be achieved without using TPPDB under solvent-free conditions; although the reaction took longer $(4 \mathrm{~h})$ and afforded a lower yield (92\%). However, although acetophenone reacted with aniline and TMSCN using a catalytic amount of TPPDB to afford a high-yield of the desired $\alpha$-aminonitrile under solvent-free conditions at room temperature (Table 1), when this reaction was repeated without using TPPDB, the corresponding $\alpha$-aminonitrile could not be achieved even after extended periods $(4 \mathrm{~h})$. When this reaction was repeated employing 
TPPDB without using TMSCN, the corresponding imine was obtained; without TPPDB no imine was observed. This implies a role for TPPDB in the generation of the corresponding imines in situ, particularly from ketones.

Table 1 Effect of Triphenylphosphine Dibromide on the Formation of $\alpha$-Aminonitriles I

\begin{tabular}{|c|c|c|c|c|c|}
\hline $\mathrm{R}^{1}$ & $\mathrm{R}^{2}$ & $\mathrm{R}^{3}$ & Time & TPPDB (mol\%) & Yield (\%) \\
\hline $\mathrm{Ph}$ & $\mathrm{H}$ & $\mathrm{Ph}$ & $20 \mathrm{~min}$ & 10 & 98 \\
\hline $\mathrm{Ph}$ & $\mathrm{H}$ & $\mathrm{Ph}$ & $4 \mathrm{~h}$ & absent & 92 \\
\hline $\mathrm{Ph}$ & $\mathrm{Me}$ & $\mathrm{Ph}$ & $25 \mathrm{~min}$ & 10 & 98 \\
\hline $\mathrm{Ph}$ & $\mathrm{Me}$ & $\mathrm{Ph}$ & $4 \mathrm{~h}$ & absent & - \\
\hline
\end{tabular}

Comparing the catalytic activity of TPPBD with some reported catalysts such as $\mathrm{I}_{2}$, guanidine hydrochloride, and cellulose sulfuric acid for the synthesis of $\alpha$-aminonitriles under solvent-free conditions, it was found that TPPDB was superior, achieving high yields of the desired products in shorter reaction times (Table 2).

Table 2 Effect of Catalysts on the Formation of $\alpha$-Aminonitriles I

\begin{tabular}{llllll}
\hline $\mathrm{R}^{1}$ & $\mathrm{R}^{2}$ & $\mathrm{R}^{3}$ & Time (h) & Catalyst (10 mol\%) & Yield (\%) \\
\hline $\mathrm{Ph}$ & $\mathrm{H}$ & $\mathrm{Ph}$ & 4 & $\mathrm{I}_{2}$ & 58 \\
$\mathrm{Ph}$ & $\mathrm{Me}$ & $\mathrm{Ph}$ & 8 & $\mathrm{I}_{2}$ & 52 \\
$\mathrm{Ph}$ & $\mathrm{H}$ & $\mathrm{Ph}$ & 2 & $\mathrm{GuHCl}$ & 78 \\
$\mathrm{Ph}$ & $\mathrm{Me}$ & $\mathrm{Ph}$ & 4 & GuHCl & 64 \\
$\mathrm{Ph}$ & $\mathrm{H}$ & $\mathrm{Ph}$ & 2 & cellulose sulfuric acid & 66 \\
$\mathrm{Ph}$ & $\mathrm{Me}$ & $\mathrm{Ph}$ & 4 & cellulose sulfuric acid & 53 \\
$\mathrm{Ph}$ & $\mathrm{H}$ & $\mathrm{Ph}$ & $25 \mathrm{~min}$ & TPPBD & 98 \\
$\mathrm{Ph}$ & $\mathrm{Me}$ & $\mathrm{Ph}$ & 30 min & TPPBD & 98 \\
\hline
\end{tabular}

The scope of this reaction was further explored with a range of aliphatic and aromatic substituted aldehydes and ketones bearing electron-releasing and electron-withdrawing functionalities and primary aliphatic and aromatic amines having electron-releasing and electronwithdrawing functional groups. Best yields of the products were obtained when an electron-releasing group was present at the para-position of the aromatic aldehydes, ketones, and amines (Table 3 ).

Table 3 Synthesis of $\alpha$-Aminonitriles of General Formula I

\begin{tabular}{lllllll}
\hline Entry $\mathrm{R}^{1}$ & $\mathrm{R}^{2}$ & $\mathrm{R}^{3}$ & \multicolumn{2}{c}{$\begin{array}{l}\text { Time } \\
(\mathrm{min})\end{array}$} & $\begin{array}{l}\text { Yield Ref. } \\
(\%)\end{array}$ \\
\hline 1 & $\mathrm{Ph}$ & $\mathrm{H}$ & $\mathrm{Ph}$ & 25 & 98 & $8 \mathrm{~d}$ \\
2 & $4-\mathrm{ClC}_{6} \mathrm{H}_{4}$ & $\mathrm{H}$ & $\mathrm{Ph}$ & 25 & 97 & $8 \mathrm{~m}$ \\
3 & $4-\mathrm{O}_{2} \mathrm{NC}_{6} \mathrm{H}_{4}$ & $\mathrm{H}$ & $\mathrm{Ph}$ & 30 & 94 & $8 \mathrm{i}$ \\
4 & $4-\mathrm{BrC}_{6} \mathrm{H}_{5}$ & $\mathrm{H}$ & $\mathrm{Ph}$ & 30 & 94 & $9 \mathrm{i}$
\end{tabular}

Table 3 Synthesis of $\alpha$-Aminonitriles of General Formula I

\begin{tabular}{|c|c|c|c|c|c|c|}
\hline Entry & $\mathrm{R}^{1}$ & $\mathrm{R}^{2}$ & $\mathrm{R}^{3}$ & $\begin{array}{l}\text { Time } \\
\text { (min) }\end{array}$ & $\begin{array}{l}\text { Yield } \\
(\%)\end{array}$ & Ref \\
\hline 5 & $3,4-\mathrm{Cl}_{2} \mathrm{C}_{6} \mathrm{H}_{3}$ & $\mathrm{H}$ & $\mathrm{Ph}$ & 30 & 95 & $9 \mathrm{i}$ \\
\hline 6 & $4-\mathrm{FC}_{6} \mathrm{H}_{4}$ & $\mathrm{H}$ & $\mathrm{Ph}$ & 35 & 91 & $9 \mathrm{~b}$ \\
\hline 7 & 4- $\mathrm{MeC}_{6} \mathrm{H}_{4}$ & $\mathrm{H}$ & $\mathrm{Ph}$ & 30 & 90 & $9 \mathrm{a}$ \\
\hline 8 & $4-\mathrm{MeOC}_{6} \mathrm{H}_{4}$ & $\mathrm{H}$ & $\mathrm{Ph}$ & 35 & 91 & $9 \mathrm{a}$ \\
\hline 9 & 3-pyridyl & $\mathrm{H}$ & $\mathrm{Ph}$ & 30 & 89 & $8 \mathrm{i}$ \\
\hline 10 & $\mathrm{C}_{9} \mathrm{H}_{19}$ & $\mathrm{H}$ & $\mathrm{Ph}$ & 60 & 80 & $8 p$ \\
\hline 11 & Et & $\mathrm{H}$ & $\mathrm{Ph}$ & 60 & 89 & $8 \mathrm{q}$ \\
\hline 12 & $\mathrm{Ph}$ & $\mathrm{H}$ & 4- $\mathrm{MeC}_{6} \mathrm{H}_{4}$ & 25 & 95 & $8 \mathrm{~m}$ \\
\hline 13 & $\mathrm{Ph}$ & $\mathrm{H}$ & 4- $\mathrm{MeOC}_{6} \mathrm{H}_{4}$ & 25 & 96 & $8 p$ \\
\hline 14 & $\mathrm{Ph}$ & $\mathrm{Me}$ & $\mathrm{Ph}$ & 30 & 98 & $9 b$ \\
\hline 15 & $\mathrm{Ph}$ & $\mathrm{Me}$ & $4-\mathrm{MeOC}_{6} \mathrm{H}_{4}$ & 30 & 99 & $8 n$ \\
\hline 16 & $\mathrm{Ph}$ & $\mathrm{Me}$ & $4-\mathrm{ClC}_{6} \mathrm{H}_{4}$ & 30 & 92 & $8 \mathrm{n}$ \\
\hline 17 & $\mathrm{Ph}$ & $\mathrm{H}$ & $\mathrm{Bn}$ & 25 & 95 & $9 b$ \\
\hline 18 & 1-naphthyl & $\mathrm{H}$ & $\mathrm{Ph}$ & 30 & 93 & 80 \\
\hline 19 & 9-anthryl & $\mathrm{H}$ & $\mathrm{Ph}$ & 35 & 91 & $8 \mathrm{r}$ \\
\hline 20 & $c-\operatorname{Pr}$ & $\mathrm{H}$ & $\mathrm{Ph}$ & 30 & 90 & 80 \\
\hline 21 & $\mathrm{Ph}$ & $\mathrm{Me}$ & $\mathrm{Bn}$ & 30 & 93 & $9 \mathrm{~b}$ \\
\hline 22 & $-\left(\mathrm{CH}_{2}\right)_{4}-$ & & & $\mathrm{Ph}$ & 94 & $8 \mathrm{n}$ \\
\hline 23 & $-\left(\mathrm{CH}_{2}\right)_{5}^{-}$ & & & $\mathrm{Ph}$ & 98 & $8 \mathrm{n}$ \\
\hline 24 & $-\left(\mathrm{CH}_{2}\right)_{6}-$ & & & $\mathrm{Ph}$ & 96 & $9 \mathrm{a}$ \\
\hline 25 & $\mathrm{Ph}$ & Et & $\mathrm{Ph}$ & 30 & 92 & $9 b$ \\
\hline 26 & $\mathrm{Ph}$ & Et & $\mathrm{Bn}$ & 30 & 88 & $9 \mathrm{~b}$ \\
\hline 27 & $\mathrm{Ph}$ & $\mathrm{Ph}$ & $\mathrm{Ph}$ & 35 & 85 & $9 \mathrm{~b}$ \\
\hline 28 & 2-naphthyl & $\mathrm{Me}$ & $\mathrm{Ph}$ & 35 & 84 & $9 \mathrm{~b}$ \\
\hline 29 & $i-\operatorname{Pr}$ & $\mathrm{Me}$ & $\mathrm{Ph}$ & 45 & 82 & $9 b$ \\
\hline 30 & $\mathrm{PhCH}=\mathrm{CH}$ & $\mathrm{H}$ & $\mathrm{Ph}$ & 45 & 80 & $9 \mathrm{~b}$ \\
\hline 31 & $\mathrm{Ph}$ & $\mathrm{H}$ & $n$-Bu & 35 & 81 & $8 \mathrm{i}$ \\
\hline 32 & 4- $\mathrm{MeC}_{6} \mathrm{H}_{4}$ & $\mathrm{H}$ & $n$-Bu & 35 & 83 & - \\
\hline 33 & $c$-Hex & $\mathrm{H}$ & $\mathrm{Bn}$ & 35 & 81 & - \\
\hline 34 & $c$-Hex & $\mathrm{H}$ & $\mathrm{Ph}$ & 35 & 80 & - \\
\hline 35 & $n-\mathrm{Bu}$ & $n-\mathrm{Bu}$ & $\mathrm{Ph}$ & 45 & 86 & $8 \mathrm{r}$ \\
\hline 36 & 2-naphthyl & $\mathrm{Ph}$ & $\mathrm{Ph}$ & 50 & 83 & $8 \mathrm{r}$ \\
\hline 37 & $4-\mathrm{O}_{2} \mathrm{NC}_{6} \mathrm{H}_{4}$ & $\mathrm{Me}$ & $\mathrm{Ph}$ & 55 & 81 & $9 b$ \\
\hline 38 & 3-Me-2- $\mathrm{HSC}_{6} \mathrm{H}_{3}$ & $\mathrm{Me}$ & $\mathrm{Ph}$ & 55 & 83 & 80 \\
\hline 39 & 3-pyridyl & $\mathrm{Me}$ & $4-\mathrm{MeOC}_{6} \mathrm{H}_{4}$ & 45 & 87 & $8 \mathrm{n}$ \\
\hline 40 & $3,4-\left(\mathrm{OCH}_{2} \mathrm{O}\right) \mathrm{C}_{6} \mathrm{H}_{3}$ & $\mathrm{Me}$ & $4-\mathrm{MeOC}_{6} \mathrm{H}_{4}$ & 50 & 82 & $8 \mathrm{n}$ \\
\hline
\end{tabular}


In conclusion, we have developed a simple and efficient method for the synthesis of $\alpha$-aminonitriles starting from their corresponding carbonyl compounds, amines, and trimethylsilyl cyanide, by employing a catalytic amount of TPPDB under solvent-free conditions.

\section{Acknowledgment}

The authors wish to thank the Pro-Vice Chancellor, and Dean, Research (Science and Technology) of Amity University, Lucknow for his constant encouragement and support.

\section{References and Notes}

(1) (a) Undavia, N. K.; Patwa, B. S.; Navadiya, H. D.; Jivani, A. R.; Dave, P. N. Int. J. Chem. Sci. 2009, 7, 1019. (b) Mantri, M.; de Graaf, O.; van Veldhoven, J.; Goeblyoes, A.; von Frijtag Drabbe Künzel, J. K.; Mulder-Krieger, T.; Link, R.; de Vries, H.; Beukers, M. W.; Brussee, J.; Ijzerman, A. P. J. Med. Chem. 2008, 51, 4449. (c) Loeser, R.; Schilling, K.; Dimmig, E.; Guetschow, M. J. Med. Chem. 2005, 48, 7688. (d) Ward, Y. D.; Thomson, D. S.; Frye, L. L.; Cywin, C. L.; Morwick, T.; Emmanuel, M. J.; Zindell, R.; McNeil, D.; Bekkali, Y.; Giradot, M.; Hrapchak, M.; DeTuri, M.; Crane, K.; White, D.; Pav, S.; Wang, Y.; Hao, M.-H.; Grygon, C. A.; Labadia, M. E.; Freeman, D. M.; Davidson, W.; Hopkins, J. L.; Brown, M. L.; Spero, D. M. J. Med. Chem. 2002, 45, 5471.

(2) (a) N'ajera, C.; Sansano, J. M. Chem. Rev. 2007, 107, 4584. (b) Zuend, S. J.; Coughlin, M. P.; Lalonde, M. P.; Jacobsen, E. N. Nature 2009, 461, 968.

(3) (a) Groger, H. Chem. Rev. 2003, 103, 2795. (b) Shafran, Y. M.; Bakulev, V. A.; Mokrushin, V. S. Russ. Chem. Rev. 1989, 58, 148. (c) Michaux, J.; Niel, G.; Campagne, J.-M. Chem. Soc. Rev. 2009, 38, 2093.

(4) (a) Matier, W. L.; Owens, D. A.; Comer, W. T.; Deitchman, D.; Ferguson, H.; Seidehamel, R. J.; Young, J. R. J. Med. Chem. 1973, 16, 901. (b) Ohfune, Y.; Shinada, T. Eur. J. Org. Chem. 2005, 5127. (c) Friestad, G. K.; Mathies, A. K. Tetrahedron 2007, 63, 2541. (d) Connon, S. J. Angew. Chem. Int. Ed. 2008, 47, 1176.

(5) (a) Enders, D.; Shilvock, J. P. Chem. Soc. Rev. 2000, 29, 359. (b) Gembus, V.; Janvier, S.; Lecouve, J.-P.; Gloanec, P.; Marsais, F.; Levacher, V. Eur. J. Org. Chem. 2010, 3583. (c) Yin, B.; Zhang, Y.; Xu, L.-W. Synthesis 2010, 3583.

(6) (a) Strecker, A. Justus Liebigs Ann. Chem. 1850, 75, 27. (b) Merino, P.; Marques-Lopez, E.; Tejero, T.; Herrera, R. P. Tetrahedron 2009, 65, 1219. (c) Pastori, N.; Gambarotti, C.; Punta, C. Mini-Rev. Med. Chem. 2009, 6, 184. (d) Shibasaki, M.; Kanai, M.; Mita, T. Org. React. 2008, 70, 1. (e) Wang, J.; Liu, X.; Feng, X. Chem. Rev. 2011, 111, 6947.

(7) (a) Kobayashi, S.; Ishitani, H. Chem. Rev. 1999, 99, 1069. (b) Bhanu Prasad, B. A.; Bisai, A.; Singh, V. K. Tetrahedron Lett. 2004, 45, 9565. (c) Harusawa, S.; Hamada, Y.; Shioiri, T. Tetrahedron Lett. 1979, 4663. (d) Nakamura, S.; Sato, N.; Sugimoto, M.; Toru, T. Tetrahedron: Asymmetry 2004, 15, 1513. (e) Kantam, M. L.; Mahendar, K.; Sreedhar, B.; Choudary, B. M. Tetrahedron 2008, 64, 3351. (f) Li, Z.; Ma, Y.; Xu, J.; Shi, J.; Cai, H. Tetrahedron Lett. 2010, 51, 3922. (g) Cruz-Acosta, F.; Santos-exposito, A.; Armas, P.; GarciaTellado, F. Chem. Commun. 2009, 6839. (h) Sipos, S.; Jablonkai, I. Tetrahedron Lett. 2009, 50, 1844. (i) Abell, J. P.; Yamamoto, H. J. Am. Chem. Soc. 2009, 131, 15118. (j) Enders, D.; Shilvock, J. P. Chem. Soc. Rev. 2000, 29, 359.

(8) (a) De , S. K. J. Mol. Catal. A: Chem. 2005, 225, 169. (b) North, M. Angew. Chem. Int. Ed. 2004, 43, 4126. (c) Murahashi, S. I.; Komia, N.; Terai, H.; Nakae, T. J. Am. Chem. Soc. 2003, 125, 15312. (d) Ranu, B. C.; Dey, S. S.; Hajra, A. Tetrahedron 2002, 58, 2529. (e) Shen, Z. L.; Ji, S. J.; Loh, T. P. Tetrahedron 2008, 64, 8159. (f) Narasimhulu, M.; Reddy, T. S.; Mahesh, K. C.; Reddy, S. M.; Reddy, A. V.; Venkateshwarlu, Y. J. Mol. Catal. A: Chem. 2007, 264, 288. (g) De, S. K.; Gibbs, R. A. Tetrahedron Lett. 2004, 45, 7407. (h) Royer, L.; De, S. K.; Gibbs, R. A. Tetrahedron Lett. 2005, 46, 4595. (i) Karmakar, B.; Banerji, J. Tetrahedron Lett. 2010, 51, 2748. (j) Mojtahedi, M. M.; Abaee, S.; Alishiri, T. Tetrahedron Lett. 2009, 50, 2322. (k) Paraskar, S.; Sudalai, A. Tetrahedron Lett. 2006, 47, 5759. (1) Kobayashi, S.; Busujima, T.; Nagayama, S. Chem. Commun. 1998, 981. (m) Martinez, R.; Ramon, D. J.; Yus, M. Tetrahedron Lett. 2005, 46, 8471. (n) Zhang, G. W.; Zheng, D. H.; Nie, J.; Wang, T.; Ma, J. A. Org. Biomol. Chem. 2010, 8, 1399. (o) Ramesh, S.; Shivakumar, K.; Panja, C.; Arunanchalam, P. N.; Lalitha, A. Synth. Commun. 2010, 40, 3544. (p) Paraskar, A. S.; Sudalai, A. Tetrahedron Lett. 2006, 47, 5759. (q) Li, Z.; Ma, Y.; Xu, J.; Shi, J.; Cai, H. Tetrahedron Lett. 2010, 51, 3922. (r) Wang, J.; Masui, Y.; Onaka, M. Eur. J. Org. Chem. 2010, 1763.

(9) (a) Hajipour, A. R.; Ghayeb, Y.; Sheikhan, N. J. Iran. Chem. Soc. 2010, 7, 447. (b) Khan, N. H.; Agrawal, S.; Kureshy, R. I.; Abdi, S. H. R.; Singh, S.; Suresh, E.; Jasra, R. V. Tetrahedron Lett. 2008, 49, 640. (c) Rafiee, E.;

Rashidzadeh, S.; Joshaghani, M.; Chalabeh, H.; Afza, K. Synth. Commun. 2008, 38, 2741. (d) Kantam, M. L.;

Mahender, K.; Sreedhar, B.; Choudhary, B. M. Tetrahedron 2008, 64, 3351. (e) Oskooie, H. A.; Heravi, M. M.; Sadnia, A.; Jannati, F.; Behbahani, F. K. Monatsh. Chem. 2008, 139, 27. (f) Arefi, H. A.; Khaksar, S.; Shiroodi, R. K. J. Mol. Catal. A: Chem. 2007, 271, 142. (g) Shaabani, A.; Maleki, A. Appl. Catal., A 2007, 331, 149. (h) Yadav, J. S.; Reddy, B. V. S.; Eeshwarain, B.; Srinivas, M. Tetrahedron 2004, 60 , 1767. (i) Rafiee, E.; Rashidzadeh, S.; Azad, A. J. Mol. Catal. A: Chem. 2007, 261, 49. (j) Yadav, J. S.; Reddy, B. V. S.; Eshwaraiah, B.; Srinivas, M.; Vishnumurthy, P. New J. Chem. 2003, 27, 462. (k) De, S. K. Synth. Commun. 2005 , 35, 1577. (l) Shaabani, A.; Maleki, A.; Soudi, M. R.; Mofakham, H. Catal. Commun. 2009, 10, 945. (m) Niknam, K.; Saberi, D.; Sefat, M. N. Tetrahedron Lett. 2010, 51, 2959. (n) Kazemeini, A.; Azizi, N.; Saidi, M. R. Russ. J. Org. Chem. 2006, 42, 48. (o) Jarusiewicz, J.; Choe, Y.; Yoo, K. S.; Park, C. P.; Jung, K. W. J. Org. Chem. 2009, 74, 2873. (p) Karimi, B.; Maleki, A.; Elhamifar, D.; Clark, J. H.; Hunt, A. J. Chem. Commun. 2010, 46, 6947. (q) Khan, N. H.; Saravanan, S.; Kureshy, R. I.; Abdi, S. H. R.; Bajaj, H. C. Tetrahedron: Asymmetry 2010, 21, 2076. (r) Sudhakar, D.; Rao, V. M.; Suresh, M.; Rao, C. V. J. Chem. Res. 2010, 34, 12. (s) Karimi, B.; Maleki, A. Chem. Commun. 2009, 5180.

(10) (a) Garima Synlett 2010, 1426. (b) Heck, M.-P.; Matt, C.; Wagner, A.; Toupet, L.; Mioskowski, C. Eur. J. Org. Chem. 2010, 966. (c) Salome, C.; Kohn, H. Tetrahedron 2009, 65, 456. (d) Bressy, C.; Alberico, D.; Lautens, M. J. Am. Chem. Soc. 2005, 127, 13148. (e) Bartley, D. M.; Coward, J. K. J. Org. Chem. 2005, 70, 6757. (f) Dormoy, J.-R.; Castro, B. eEROS Encyclopedia of Reagents for Organic Synthesis 2001.

(11) (a) Chaturvedi, D.; Kumar, A.; Ray, S. Tetrahedron Lett. 2003, 44, 7637. (b) Chaturvedi, D.; Ray, S. Tetrahedron Lett. 2006, 47, 1307. (c) Chaturvedi, D.; Ray, S. Tetrahedron Lett. 2007, 48, 149. (d) Chaturvedi, D.; Mishra, N.; Mishra, V. Tetrahedron Lett. 2007, 48, 5043. (e) Chaturvedi, D.; Mishra, N.; Mishra, V. Synthesis 2008, 355. (f) Chaturvedi, D.; Chaturvedi, A. K.; Mishra, N.; Mishra, V. Tetrahedron Lett. 2008, 49, 4886. 
(g) Chaturvedi, A. K.; Chaturvedi, D.; Mishra, N.; Mishra, V. J. Iran. Chem. Soc. 2010, 7, 702. (h) Chaturvedi, A. K.; Chaturvedi, D.; Mishra, N.; Mishra, V. J. Iran. Chem. Soc. 2011, 8, 396.

(12) Synthesis of $\alpha$-Aminonitriles; Typical Procedure: A mixture of aldehyde $(1 \mathrm{mmol})$, amine $(1 \mathrm{mmol})$, triphenylphosphine dibromide (10 $\mathrm{mol} \%)$, and trimethylsilylcyanide $(1.2 \mathrm{mmol})$ was stirred at room temperature until the reaction was complete (monitored by TLC). The reaction mixture was then extracted with EtOAc $(\times 3)$, dried over anhydrous $\mathrm{Na}_{2} \mathrm{SO}_{4}$, filtered, and concentrated. Purification of the crude product by chromatography on silica gel (60-120 mesh; petroleum ether-EtOAc, 5:1) gave the pure product.

2-Anilino-2-phenylacetonitrile (Table 3, Entry 1): Lightyellow solid; $\mathrm{mp} 85-86^{\circ} \mathrm{C}$; IR $\left(\mathrm{CHCl}_{3}\right): 3368,3055,2233$, $1602,1502 \mathrm{~cm}^{-1} ;{ }^{1} \mathrm{H}$ NMR $\left(300 \mathrm{MHz}, \mathrm{CDCl}_{3}\right): \delta=4.03(\mathrm{~d}$, $J=9 \mathrm{~Hz}, 1 \mathrm{H}), 5.41(\mathrm{~d}, J=9 \mathrm{~Hz}, 1 \mathrm{H}), 6.76(\mathrm{~d}, J=9 \mathrm{~Hz}$, $2 \mathrm{H}), 6.90(\mathrm{t}, J=6 \mathrm{~Hz}, 1 \mathrm{H}), 7.30(\mathrm{t}, J=9 \mathrm{~Hz}, 2 \mathrm{H}), 7.44(\mathrm{~m}$, $3 \mathrm{H}), 7.59(\mathrm{~m}, 2 \mathrm{H}) ;{ }^{13} \mathrm{C}$ NMR $\left(75 \mathrm{MHz}, \mathrm{CDCl}_{3}\right): \delta=49.8$, 114.0, 118.1, 119.9, 127.0, 128.3, 129.4, 129.8, 133.6, 144.6; MS (ESI): $m / z=208.2[\mathrm{M}]^{+}$; Anal. Calcd for
$\mathrm{C}_{14} \mathrm{H}_{12} \mathrm{~N}_{2}: \mathrm{C}, 80.74 ; \mathrm{H}, 5.81 ; \mathrm{N}, 13.45$. Found: $\mathrm{C}, 80.80 ; \mathrm{H}$, 5.76; N, 13.47.

2-Anilino-2-(4-chlorophenyl)acetonitrile (Table 3, Entry 2): White solid; $\mathrm{mp} 96-98^{\circ} \mathrm{C}$; IR $\left(\mathrm{CHCl}_{3}\right): 3365,3055$, 2235, 1603, $1504 \mathrm{~cm}^{-1} ;{ }^{1} \mathrm{H} \mathrm{NMR}\left(300 \mathrm{MHz}, \mathrm{CDCl}_{3}\right)$ : $\delta=$ $4.02(\mathrm{~d}, J=6 \mathrm{~Hz}, 1 \mathrm{H}), 5.41(\mathrm{~d}, J=9 \mathrm{~Hz}, 1 \mathrm{H}), 6.75(\mathrm{~d}, J=$ $9 \mathrm{~Hz}, 2 \mathrm{H}), 6.92(\mathrm{t}, J=6 \mathrm{~Hz}, 1 \mathrm{H}), 7.28(\mathrm{~m}, 2 \mathrm{H}), 7.42(\mathrm{~d}, J$ $=9 \mathrm{~Hz}, 2 \mathrm{H}), 7.53(\mathrm{~d}, J=6 \mathrm{~Hz}, 2 \mathrm{H}) ;{ }^{13} \mathrm{C}$ NMR $(75 \mathrm{MHz}$, $\left.\mathrm{CDCl}_{3}\right): \delta=49.5,114.2,117.8,120.4,128.4,129.2,129.6$, 132.8, 135.4, 144.3; MS (ESI): $m / z=242.1[\mathrm{M}]^{+}$; Anal. Calcd for $\mathrm{C}_{14} \mathrm{H}_{11} \mathrm{ClN}_{2}$ : C, 69.28; H, 4.57; N, 11.54; Found: $\mathrm{C}, 69.19 ; \mathrm{H}, 4.63 ; \mathrm{N}, 11.56$.

2-Anilino-2-(4-nitrophenyl)acetonitrile (Table 3, Entry 3): Gummy solid; IR $\left(\mathrm{CHCl}_{3}\right): 3381,3063,2225,1601$, 1550, $1502 \mathrm{~cm}^{-1} ;{ }^{1} \mathrm{H}$ NMR (300 MHz, $\left.\mathrm{CDCl}_{3}\right): \delta=4.08(\mathrm{~d}$, $J=9 \mathrm{~Hz}, 1 \mathrm{H}), 5.57(\mathrm{~d}, J=9 \mathrm{~Hz}, 1 \mathrm{H}), 6.68(\mathrm{~d}, J=9 \mathrm{~Hz}$, $2 \mathrm{H}), 6.78(\mathrm{t}, J=8 \mathrm{~Hz}, 1 \mathrm{H}), 7.29(\mathrm{t}, J=9 \mathrm{~Hz}, 2 \mathrm{H}), 7.8(\mathrm{~d}, J$ $=9 \mathrm{~Hz}, 2 \mathrm{H}), 8.1(\mathrm{~d}, J=9 \mathrm{~Hz}, 2 \mathrm{H}) ;{ }^{13} \mathrm{C}$ NMR $(75 \mathrm{MHz}$, $\left.\mathrm{CDCl}_{3}\right): \delta=49.8,115.3,118.0,127.0,127.7,127.8,128.6$, 129.0, 133.8, 144.1, 145.0; MS (ESI): $m / z=276.2[\mathrm{M}+$ $\mathrm{Na}]^{+}$; Anal. Calcd for $\mathrm{C}_{14} \mathrm{H}_{11} \mathrm{~N}_{3} \mathrm{O}_{2}: \mathrm{C}, 66.40 ; \mathrm{H}, 4.38 ; \mathrm{N}$, 16.59; Found: C, 66.46; H, 4.40; N, 16.51 . 